\title{
Colorectal signet ring cell carcinoma coexisting with tuberculosis
}

\author{
A Pradhan ${ }^{1}, \mathrm{~S} \mathrm{Karki}^{1}, \mathrm{~S}$ Khaniya ${ }^{2}$ \\ ${ }^{1}$ Department of Pathology and ${ }^{2}$ Department of Surgery \\ BP Koirala Institute of Health Sciences, Dharan, Nepal
}

\begin{abstract}
Background: Though tuberculosis and carcinoma of the bowel are fairly common conditions, the sites of predilection for the two differ, tuberculosis being common in the ileum while carcinoma in the large bowel. Both occurring at the same site is a very rare entity and very few cases have been reported in the literature and none has been recorded in the eastern part of Nepal. Case description: A 35 years old female presented with the complaint of constipation and passage of blood mixed stool since one month. Proctoscopy revealed constricting lesion in the rectum. Provisional diagnosis of carcinoma rectum was made. Histopathological examination showed ulceroinfiltrative lesion in the rectum measuring $11 \mathrm{~cm}$ in length and extending upto the distal end of sigmoid colon. After microscopic examination a diagnosis of Signet ring cell carcinoma of rectum and sigmoid colon coexisting with tuberculosis was made. Seven lymph nodes dissected from the specimen showed metastatic tumor deposits along with coexisting tuberculous lymphadenitis. Special stain for AFB (TB) and IHC for mucicarmine was positive.
\end{abstract}

\section{Introduction}

Developing countries like Nepal are still facing gastrointestinal tuberculosis as a major health problem. Gastrointestinal tract is the sixth most frequent site of extrapulmonary involvement by tuberculosis, ileocaecal region being the most common site. Carcinoma of bowel is also a fairly common condition but colorectal Signet Ring cell carcinoma accounts for only $1 \%$ of primary large bowel carcinoma and is associated with a poorer prognosis.

Concomitant existence of these two conditions is rare and the sites of predilection for the two also differ. Very few cases of adenocarcinoma of intestine, mostly being Mucinous, coexisting with tuberculosis are reported in the literature and none has been recorded in the eastern part of Nepal. But cases of

Address for correspondence

Dr Anju Pradhan

Associate Professor, Department of Pathology

BP Koirala Institute of Health Sciences, Dharan

Email: dranjupradhan@yahoo.com co-existing Signet ring Cell carcinoma with tuberculosis have not been reported yet.

There has been a discussion regarding the etiopathogenetic relationship of the co-existence but the true mechanism been a dilemma so far.

We are presenting this case for its rarity. Colorectal primary signet ring cell carcinoma is a rare entity; colorectal area is a rare site for intestinal tuberculosis and the coexistence of these entities is the rarest of all.

The discussion regarding the etiopathogenetic relationship of this coexistence is still debatable.

\section{Case report}

A 35 years old female presented in surgical OPD with the complaint of constipation and passage of blood mixed stool since nine month associated with significant weight loss. Proctoscopy revealed constricting lesion in the rectum. CT scan revealed a constricting lesion in the rectum with thickened wall. Provisional diagnosis of carcinoma rectum was made. 


\section{Gross}

Resected segment of large intestine was received comprising of sigmoid colon and rectum measuring together $34 \mathrm{cms}$ in total length. An ulceroinfiltrative growth was identified at the distal end of sigmoid colon reaching upto the rectum but sparing $2 \mathrm{~cm}$ of the distal end of rectum. The involved bowel wall was thickened.

Twenty three lymph nodes were dissected from the corresponding mesentery.

\section{Microscopy}

Multiple sections examined from the ulceroinfiltrative growth revealed signet ring cells arising from the mucosa and infiltrating diffusely through the submucosa into the muscle layer upto the serosa and mesentery. No glandular formations or extracellular mucin were observed. Adjacent to the tumor cells there were presence of coexisting epithelioid cell granulomas, Langhans giant cells, caseous necrosis and surrounding lymphocytes.

Seven out of total twenty three lymph nodes dissected from the specimen showed metastatic tumor deposits along with coexisting epithelioid cell granulomas, Langhans giant cells and few with caseous necrosis. Special stain for AFB (TB) was positive in the sections from intestine as well as in the lymph node. Mucicarmine was positive for the intracellular mucin present in the signet ring cells.

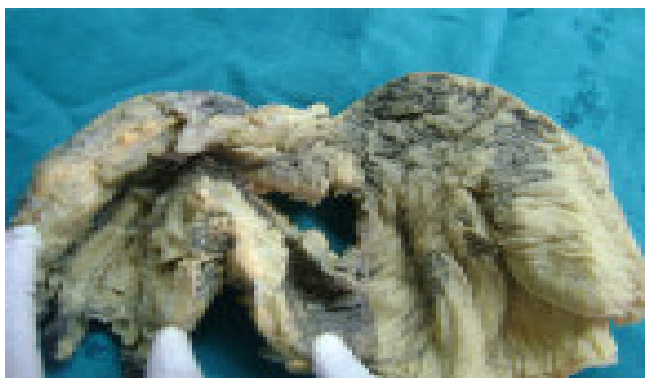

Fig 1. Gross picture revealing an ulceroinfiltrative lesion in the distal end of sigmoid colon measuring $11 \mathrm{~cm}$ in length and extending upto the rectum.

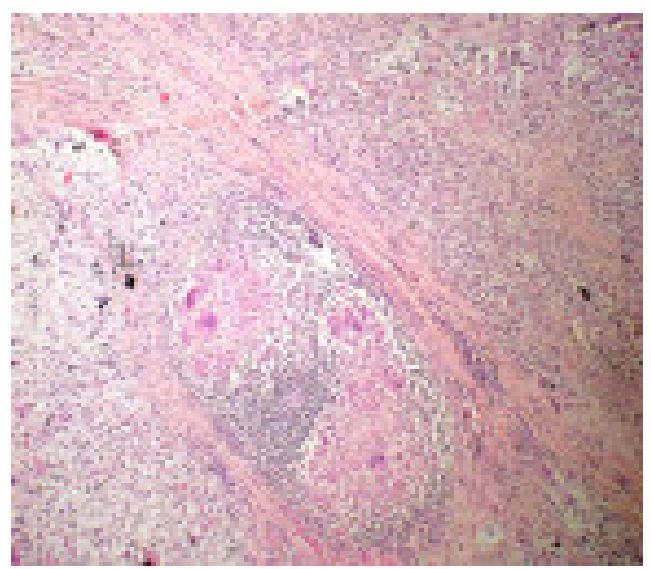

Fig. 2. Microphotograph revealing presence of signet ring cells with coexisting epithelioid cell granulomas, splitting the mucularis propria. ( $H \&$ E stain, $10 \mathrm{X}$ )

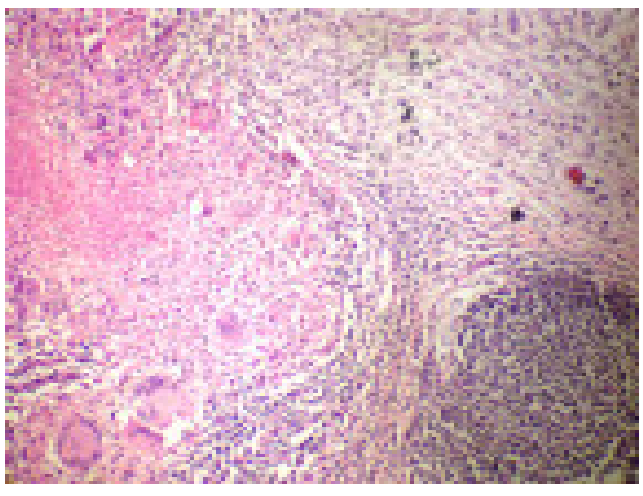

Fig 3. High power view revealing caseating epithleioid cell granuloma along with presence of diffuse signet ring cells. $H \& E$ stain, $40 X$

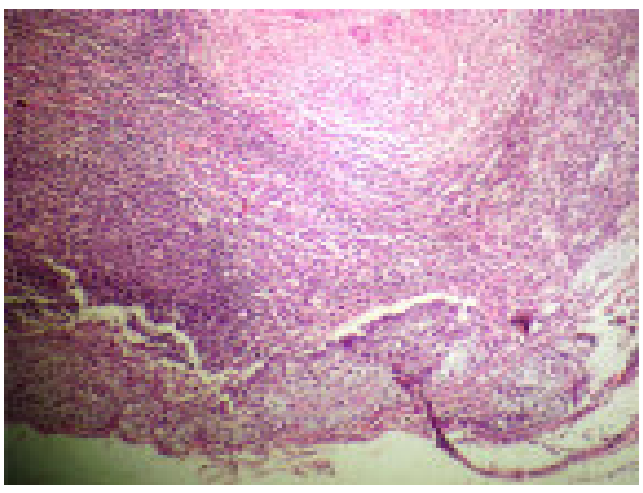

Fig. 4. Microphotograph revealing infiltration of the subcapsular sinus of a lymph node by signet ring cells.

There is presence of caseating granuloma with Langhans giant cells in the cortex. 


\section{Discussion}

Carcinoma of bowel is a common condition with colon and rectum being 40 to 60 times more commonly involved than the small bowel. Colorectal Signet Ring cell carcinoma accounts for only $1 \%$ of primary large bowel carcinoma and is associated with a poorer prognosis.

Approximately $50 \%$ of all carcinomas arise in the rectosigmoid area. In our case, tumor was seen in the distal sigmoid colon extending to the proximal rectum. Most of the reported cases have shown a predominance of right side of colon involved by the tumor in contrast to our case and none has been reported at the colorectal area. Cancer of the left colon occurs more frequently in the females under the age of 50 years. Our case also was a female patient of 35years which is similar to the findings of other authors.

Ileocaecal region is the most common site for intestinal tuberculosis, possibly owing to its abundance of lymphoid tissue, increased physiological stasis and decreased digestive activity at this site. But our case showed presence of tuberculosis at the site of presence of carcinoma, the colorectum, which is a very rare site for the occurrence for tuberculosis. Segmental or isolated colonic tuberculosis referring to involvement of the colon without ileocaecal region constitutes 9.2 per cent of all cases of abdominal tuberculosis.

Grossly, colorectal carcinoma can be either polypoidal or ulceroinfiltrative. Intestinal tuberculosis can be ulcerative, hypertrophic or ulcerohypertrophic. The tumor in our case has an ulceroinfiltrative growth with hypertrophied wall.

Signet ring cell carcinoma of colon is rare form (1\%) of primary colorectal malignancy that usually affects young patients and has worse prognosis than mucinous carcinoma. Microscopically, it is composed of signet ring cells having eccentrically pushed nucleus due to abundant intracellular mucin and the tumor grows diffusely. Our case also showed a diffuse infiltrative pattern of growth of the tumor composed of signet ring cells extending upto the serosa and mesentery. Ulceroinfiltrative carcinomas have a greater tendency for stromal invasion with lymphovasular spread.
Colorectal area is a rare site for occurrence of Tuberculosis, ileocaecal region being more common site. Our case showed concomitant presence of epithelioid cell granulomas, Langhans giant cells and caseous necrosis with the tumor.

Though the coexistence of these entities has been reported in the literature, coexistence in the mesenteric lymph node of the same case or separately has not been observed. Our case shows not only the coexistence of these entities but also their spread to the mesenteric lymph nodes and concomitant existence in it.

Colorectal carcinoma usually presents with rectal bleeding, change in bowel habits like diarrhea alternating with constipation and pain abdomen. Manifestations of colonic tuberculosis include fever, anorexia, weight loss and change in bowel habits. Pain abdomen is the predominant symptom and hematochezia occurs in less than one third. Patient in our case presented had a history of significant weight loss and presented with pain abdomen and rectal bleeding.

The etiopathological relationship of this dual pathology has always been a dilemma and many authors have discussed about it. Some consider carcinoma as a primary lesion while other suspect tuberculous ulceration and fibrosis to be carcinogenesis as stated by Maruta $\mathrm{K}$ et al. The exact mechanism has yet to be studied to reveal and raise the curtain of this coexistence. Since primary signet ring cell carcinoma is itself rare and colorectal area is a rare site for tuberculosis, in our case, we consider this as simply a coincidence.

This is a rare case of signet ring cell carcinoma and tuberculosis coexisting at the same site. Though coexistence of other types of bowel carcinoma with tuberculosis has been reported so far, coexistence of Signet Ring Cell carcinoma with tuberculosis has not yet been reported.

A definite diagnosis can be established only by histopathological evaluation which helps in proper treatment of the patient. 


\section{Summary}

This is a rare case of Adenocarcinoma and tuberculosis coexisting at the same site. Signet ring cell carcinoma of colon is rare form of colorectal malignancy that usually affects young patients. Colorectal area is a rare site for occurrence of Tuberculosis. A definite diagnosis can be established only by histopathological evaluation which helps in proper treatment of the patient.

\section{References}

1. Husain M, Sachan P, Khan S, Chauhan N, Kishore S. Co-existing abdominal tuberculosis and colon carcinoma: Case report with review of literature. Internet J Surg. 2007; 13:2.

2. Sushama Desai, Sunil Jagtap, Hemant Janugade: Coexisting Tuberculosis and Mucinous carcinoma of Caecum - A case report. MJAFI 2005; 61: 197-199.

3. Jain BK, Chandra SS, Narsimhan R, Ananthkrishnan N, Mehta RB. Coexisting tuberculosis and carcinoma of the colon. Aust N Z J Surg 1991; 61(11): 828-31.

4. Juan Rosai. Gastrointestinal tract, large bowel. In: Juan Rosai, editor. Ackerman's Surgical Pathology 8th Ed. Vol I. USA: Harcourt Brace and Company Asia Pte. Ltd; 729-799.

5. Jas JR. Tumors of the small and large intestines (including the anal region). In: D. M. Fletcher, editor. Diagnostic Histopathology of Tumors. $3^{\text {rd }}$ ed. Vol I. Elsevier; 379-416.

6. Sharma MP, Bhatia V. Abdominal Tuberculosis. Indian J Med Res 120, October 2004; 305-315.

7. Singh Y, Sayami G, Khakurel M. Coexistence of tuberculosis and Adenocarcinoma of the colon. Journal of Nepal Medical Association 2003; 42: $39-41$.
8. Chakravartty S, Chattopadyay G, Ray D, Choudhury CR, Mandal S. Concomitant Tuberculosis and Carcinoma Colon: Coincidence or Causal Nexus? Saudi J Gastroenterol. 2010 Oct-Dec; 16(4):292-4.

9. Maheshwari V, Alam K, Indu, Tyagi SP. Ileocaecal tuberculosis associated with adenocarcinoma of the caecum and colon. $\mathrm{J}$ Indian Med Assoc 1995; 93(10): 392-3. (s)

10. Tanaka K, Kondo S, Hattori F, et al. A case of colonic carcinoma associated with intestinal tuberculosis and an analysis of 26 cases reported in Japan, Gan No Rinsho 1987; 33: 1117-23. (s)

11. Sane SY, Nimbkar SA. Carcinoma colon with tuberculosis. J Postgrad Med 1980; 26930: 199200. (s)

12. Maruta K, Tanaka S, Nakamura T, Hagio S, Sato E, Nishi M. Carcinoma of the colon coexisting with tuberculosis-report of 2 cases. Gan No Rinsho. 1983 May; 29(5):448-53. 